Xiao Zhang*, Kaiyun Li and Sen Liang

\title{
Crystal structure of dimethylammonium 5-fluorouracil-1-acetate, $\mathrm{C}_{8} \mathrm{H}_{12} \mathrm{~N}_{3} \mathrm{O}_{4} \mathrm{~F}$
}
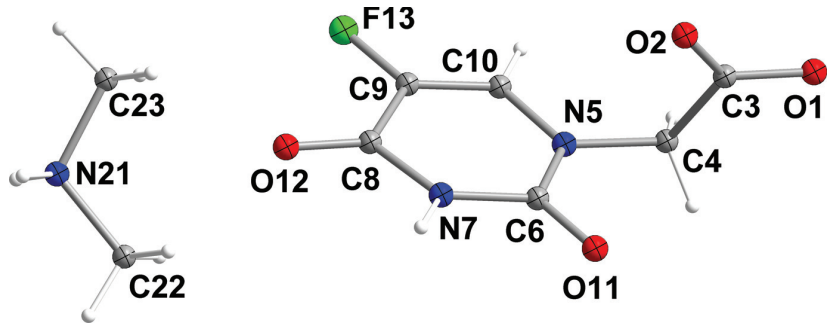

https://doi.org/10.1515/ncrs-2019-0688

Received September 12, 2019; accepted November 8, 2019;

available online December 20, 2019

\section{Abstract}

$\mathrm{C}_{8} \mathrm{H}_{12} \mathrm{~N}_{3} \mathrm{O}_{4} \mathrm{~F}$, monoclinic, $P 2_{1} / c$ (no. 14), $a=4.8090$ (3) $\AA$, $b=9.9340(6) \AA, \quad c=22.2437(13) \AA, \quad \beta=90.138(8)^{\circ}$, $V=1062.64(11) \AA^{3}, Z=4, R_{\mathrm{gt}}(F)=0.0452, w R_{\mathrm{ref}}\left(F^{2}\right)=0.1227$, $T=293(2) \mathrm{K}$.

\section{CCDC no.: 1964557}

The molecular structure is shown in the figure. Table 1 contains crystallographic data and Table 2 contains the list of the atoms including atomic coordinates and displacement

\section{Source of material}

5-Fluorouracil-1-acetic acid was obtained by the literature method [4]. 5-Fluorouracil-1-acetic acid (0.054 g, $0.3 \mathrm{mmol})$ and $\mathrm{AgBF}_{4}(0.058 \mathrm{~g}, 0.3 \mathrm{mmol})$ were dissolved in $\mathrm{CH}_{3} \mathrm{OH}$ (4 mL) under ultrasonication. After adding $0.3 \mathrm{mmol}$ phenanthroline, DMF (2 mL) was added to the mixture under ultrasonication. The mixture was sealed and heated to $70{ }^{\circ} \mathrm{C}$ for $20 \mathrm{~h}$. Then the solution was cooled to room temperature and

*Corresponding author: Xiao Zhang, School of Materials Science and Engineering, North Minzu University, No. 204 Wenchang North Street, Yin-Chuan, Ningxia 750021, P.R. China, e-mail: smile_zhang@nun.edu.cn. https://orcid.org/0000-00032162-216X

Kaiyun Li and Sen Liang: Ningxia Key Laboratory for Photovoltaic Materials, Ningxia University, No. 489 Helanshan West Road, Yin-Chuan, Ningxia 750021, P.R. China. https://orcid.org/00000003-4371-4970 (S. Liang) parameters.

Table 1: Data collection and handling.

\begin{tabular}{ll}
\hline Crystal: & Colorless needle \\
Size: & $0.17 \times 0.15 \times 0.11 \mathrm{~mm}$ \\
Wavelength: & Cu $K \alpha$ radiation $(1.54178 \AA)$ \\
$\mu:$ & $1.11 \mathrm{~mm}^{-1}$ \\
Diffractometer, scan mode: & Bruker APEX-III, $\varphi$ and $\omega$ \\
$\theta_{\text {max }}$, completeness: & $73.6^{\circ},>99 \%$ \\
$N\left(h k l_{\text {measured }}, N(h k l)_{\text {unique }}, R_{\text {int }}:\right.$ & $3999,2076,0.035$ \\
Criterion for $I_{\text {obs }}, N\left(h k l_{\text {gt }}:\right.$ & $I_{\text {obs }}>2 \sigma\left(I_{\text {obs }}\right), 1637$ \\
$N(\text { param })_{\text {refined }}:$ & 147 \\
Programs: & SHELX [1], Diamond [2], Bruker [3]
\end{tabular}

Table 2: Fractional atomic coordinates and isotropic or equivalent isotropic displacement parameters $\left(\AA^{2}\right)$.

\begin{tabular}{lrrrr}
\hline Atom & $\boldsymbol{x}$ & $\boldsymbol{y}$ & $\boldsymbol{z}$ & \multicolumn{1}{c}{$\boldsymbol{U}_{\text {iso }} \boldsymbol{U}_{\text {eq }}$} \\
\hline F13 & $0.7841(3)$ & $0.09962(12)$ & $0.49448(5)$ & $0.0267(3)$ \\
O1 & $0.3712(3)$ & $0.45061(14)$ & $0.25356(6)$ & $0.0203(3)$ \\
O2 & $0.1524(3)$ & $0.31808(13)$ & $0.32033(6)$ & $0.0194(3)$ \\
O11 & $0.1585(3)$ & $0.52371(13)$ & $0.43199(6)$ & $0.0183(3)$ \\
O12 & $0.3714(3)$ & $0.20050(14)$ & $0.57022(6)$ & $0.0223(3)$ \\
N5 & $0.5141(3)$ & $0.38024(16)$ & $0.41055(7)$ & $0.0160(4)$ \\
N7 & $0.2662(3)$ & $0.35912(16)$ & $0.49992(6)$ & $0.0157(3)$ \\
H7 & 0.1139 & 0.3837 & 0.5241 & $0.019^{*}$ \\
C3 & $0.3380(4)$ & $0.39767(19)$ & $0.30545(8)$ & $0.0162(4)$ \\
C4 & $0.5545(4)$ & $0.44330(19)$ & $0.35168(8)$ & $0.0168(4)$ \\
H4A & 0.7387 & 0.4212 & 0.3370 & $0.020^{*}$ \\
H4B & 0.5442 & 0.5403 & 0.3561 & $0.020^{*}$ \\
C6 & $0.3035(4)$ & $0.42648(18)$ & $0.44629(8)$ & $0.0150(4)$ \\
C8 & $0.4171(4)$ & $0.24957(19)$ & $0.52092(8)$ & $0.0170(4)$ \\
C9 & $0.6262(4)$ & $0.20551(19)$ & $0.47827(8)$ & $0.0181(4)$ \\
C10 & $0.6699(4)$ & $0.26921(19)$ & $0.42643(8)$ & $0.0177(4)$ \\
H10 & 0.8076 & 0.2383 & 0.4006 & $0.021^{*}$ \\
N21 & $0.1248(3)$ & $0.90736(15)$ & $0.30591(7)$ & $0.0178(4)$ \\
H21A & -0.0297 & 0.9247 & 0.2853 & $0.021^{*}$ \\
H21B & 0.2690 & 0.9292 & 0.2828 & $0.021^{*}$ \\
C22 & $0.1366(5)$ & $0.7613(2)$ & $0.31927(9)$ & $0.0270(5)$ \\
H22A & 0.3031 & 0.7417 & 0.3415 & $0.041^{*}$ \\
H22B & 0.1371 & 0.7114 & 0.2823 & $0.041^{*}$ \\
H22C & -0.0226 & 0.7361 & 0.3427 & $0.041^{*}$ \\
C23 & $0.1292(5)$ & $0.9925(2)$ & $0.36074(9)$ & $0.0223(4)$ \\
H23A & -0.0321 & 0.9735 & 0.3846 & $0.033^{*}$ \\
H23B & 0.1285 & 1.0857 & 0.3494 & $0.033^{*}$ \\
H23C & 0.2938 & 0.9733 & 0.3837 & $0.033^{*}$ \\
\hline & & & &
\end{tabular}

๖ Open Access. () 2019 Xiao Zhang et al., published by De Gruyter. (c) BY License. 
filtered, slow evaporation afforded colorless needle crystals of the title compound as an unexpected result.

\section{Experimental details}

All hydrogen atoms were positioned geometrically and allowed to ride on their parent atoms.

\section{Comment}

Pharmaceutical cocrystal compounds [5] have been extensively studied in their structures and pharmacological activity. The stability of some drug molecules will be improved after forming cocrystals with other biocompatible molecules. That's very important for drug industry. 5-Fluorouracil (5FU) and its derivatives as the first and the most important organic anticancer drugs have been widely investigated over sixty years [6]. Although our original intention is to combine some bioactive groups like 5-FU with coinage metal complexes [7-10], we fail to get the target silver complex. Only salt title compound has been obtained and is reported here. The dimethylammonium part of the title compound deduces from the decomposition of DMF [11].

The title compound has four anions and four cations in the unit cell. The bond lengths and angles are in the normal ranges [4]. Similar to bis(4-dimethylamino-pyridin-1ium) tetrafluorosuccinate [12], the 5-fluorouracil-1-acetate and dimethylammonium form strong $\mathrm{N}-\mathrm{H} \cdots \mathrm{O}$ hydrogen bonds. The adjacent 5-FU groups are forming 5-FU and 5-FU mismatch basepairs via $\mathrm{N}-\mathrm{H} \cdots \mathrm{O}$ duplicate hydrogen bonds. This observation is rarely reported in the crystal structures of 5-FU derivatives.

Acknowledgements: This work was financially supported by the Key Scientific Research Projects in 2017 at North Minzu University (No. 2017KJ07).

\section{References}

1. Sheldrick, G. M.: A short history of SHELX. Acta Crystallogr. A64 (2008) 112-122.

2. Brandenburg, K.: DIAMOND. Visual Crystal Structure Information System. Version 3.2i. Crystal Impact, Bonn, Germany (2012).

3. Bruker. APEX3 and SAINT. Bruker AXS Inc., Madison, WI, USA (2012).

4. Hu, M. L.; Yuan, J. X.; Morsali, A.: La"l' complexes of uracil1-acetic acid, $\left[\text { La(phen) } X_{3}\right]_{n}, X=$ uracil-1-acetate (UA) and 5-fluorouracil-1-acetate (5-FUA), two 2D coordination polymer, structural and thermal studies. Solid State Sci. 8 (2006) 981-987.

5. Qiao, N.; Li, M.; Schlindwein, W.; Malek, N.; Davies, A.; Trappitt, G.: Pharmaceutical cocrystals: an overview. Int. J. Pharm. 419 (2011) 1-11.

6. Heidelberger, C.; Chaudhuri, N. K.; Danneberg, P.; Mooren, D.; Griesbach, L.; Duschinsky, R.; Schnitzer, R. J.; Pleven, E.; Scheiner, J.: Fluorinated pyrimidines, a new class of tumour-inhibitory compounds. Nature 179 (1957) 663-666.

7. Liu, K.-G.; Rouhani, F.; Shan, Q.-D.; Wang, R.; Li, J.; Hu, M.-L.; Cheng, X.; Morsali, A.: Ultrasonic-assisted fabrication of thin-film electrochemical detector of $\mathrm{H}_{2} \mathrm{O}_{2}$ based on ferrocenefunctionalized silver cluster. Ultrason. Sonochem. 56 (2019) 305-312.

8. Li, J.-Z.; Bigdeli, F.; Gao, X.-M.; Wang, R.; Wei, X.-W.; Yan, X.-W.; Hu, M.-L.; Liu, K.-G.; Morsali, A.: Trivalent tetrahedral anion template: a 26-nucleus silver alkynyl cluster encapsulating vanadate. Inorg. Chem. 58 (2019) 5397-5400.

9. Liu, K.-G.; Shan, Q.-D.; Nie, J.; Yan, X.-W.: A 2D supramolecular network based on aromatic $\pi \cdots \pi$ stacking interactions. Z. Anorg. Allg. Chem. 644 (2018) 82-85.

10. Liu, K.-G.; Wang, R.; Han, H.-Y.; Shan, Q.-D.; Nie, J.; Yan, X.-W.: Incorporating ferrocenecarboxylato(-1) into a $\left[\mathrm{Cu}_{2}{ }_{2}(\mu-\mathrm{dppm})_{2}\right]^{2+}$ (dppm $=$ bis(diphenylphosphino)methane) scaffold for hydrogen peroxide sensing. J. Coord. Chem. 71 (2018) 3500-3509.

11. Muzart, J. N.: $N$-Dimethylformamide: much more than a solvent. Tetrahedron 65 (2009) 8313-8323.

12. Liu, K.-G.; Nie, J.: Crystal structure of bis(4-dimethylaminopyridin-1-ium) tetrafluorosuccinate, $\mathrm{C}_{18} \mathrm{H}_{22} \mathrm{~F}_{4} \mathrm{~N}_{4} \mathrm{O}_{4}$. Z. Kristallogr. NCS 233 (2018) 321-323. 